\title{
Pelvic tuberculosis mimicking ovarian cancer: a series of 13 cases
}

Baki Erdem ${ }^{1}$, Nuri Peker ${ }^{2}$, Ayse Inci ${ }^{3}$, Suat Can Ulukent ${ }^{4}$, Ipek Yildiz Ozaydin ${ }^{5}$, Ilkbal Temel Yuksel ${ }^{1}$, Volkan Ulker ${ }^{1}$, Ceyhun Numanoglu¹, Ozgur Akbayir ${ }^{1}$

\author{
'Department of Gynecologic Oncology, Kanuni Sultan Suleyman Training and \\ Research Hospital, Istanbul, Turkey \\ ${ }^{2}$ Department of Obstetrics and Gynecology, Acibadem University Atakent Hospital, \\ Istanbul, Turkey \\ ${ }^{3}$ Department of Infectious Diseases, Kanuni Sultan Suleyman Training and Research \\ Hospital, Istanbul, Turkey \\ ${ }^{4}$ Department of General Surgery, Kanuni Sultan Suleyman Training and Research, \\ Istanbul, Turkey \\ ${ }^{5}$ Department of Pathology, Kanuni Sultan Suleyman Training and Research Hospital, \\ Istanbul, Turkey
}

Submitted: 19 September 2017

Accepted: 25 November 2017

Arch Med Sci Civil Dis 2017; 2: e177-e181

DOI: https://doi.org/10.5114/amscd.2017.72541

Copyright (c) 2017 Termedia \& Banach

\section{Abstract}

Introduction: In this study, we aimed to investigate the incidence of pelvic tuberculosis mimicking ovarian cancer.

Material and methods: The data of a total of 13 patients diagnosed with peritoneal or genital tuberculosis in the gynecologic oncology unit between January 1, 2004 and January 1, 2016 were retrospectively analyzed.

Results: The mean age of the patients was $39.4 \pm 14.1$ (range: 22-67) years. The mean parity number was $1.69 \pm 1.1$ (range: $0-5$ ) and mean body mass index was $23.3 \pm 4.1$ (range: $18-43) \mathrm{kg} / \mathrm{m}^{2}$. The mean cancer antigen 125 (CA125) level was $473 \pm 394$ (range: $22-1153$ ) $\mathrm{U} / \mathrm{ml}$. The most common complaints were abdominal pain $(n=3)$ and bloating $(n=9)$. The most common ultrasonographic findings were diffuse ascites and adnexal mass. No signs of malignancy were found in 6 patients who underwent paracentesis and in 5 patients who underwent endoscopy preoperatively. Six patients underwent laparoscopic surgery, whereas 7 patients underwent laparotomy by subumbilical medial incision. Findings in abdominal examination included diffuse adhesions (13/13), diffuse ascites (11/13), pelvic mass (10/13), and diffuse nodular formations (11/13).

Conclusions: In tuberculosis-endemic regions, peritoneal tuberculosis should be considered in the differential diagnosis of malignant adnexal masses. Although various tests are helpful, histopathological and bacterial examination of tissue samples is of utmost importance for establishing a definitive diagnosis.

Key words: adnexal mass, peritonitis, tuberculosis.

\section{Introduction}

Tuberculosis (TB) is one of the major public health problems reported to be particularly endemic in developing countries [1, 2]. On average, 8.6 million cases of TB are diagnosed every year, with 1.3 million cases ending in death [1-3]. Although TB primarily affects the lungs,

\author{
Corresponding author: \\ Baki Erdem \\ Department of \\ Gynecologic Oncology \\ Kanuni Sultan \\ Suleyman Training \\ and Research Hospital \\ Istanbul, Turkey \\ E-mail: \\ drberdem@yandex.com
}


non-pulmonary involvement in sites such as the urogenital system and peritoneum has also been reported $[2,3]$.

Urogenital TB accounts for about $15 \%$ of all extragenital TB cases, and may lead to severe health problems in gynecology and obstetrics practice [2]. Urogenital system or peritoneal involvement is usually due to the homogeneous dissemination of primary pulmonary TB [2]. The most commonly involved sites in the female genital system are the fallopian tubes, followed by the endometrium $[1,2]$. Main complaints include chronic pelvic pain, menstrual irregularity, infertility, and weight loss; however, an adnexal mass, ascites, and elevated CA125 levels detected during follow-up visits may also be encountered in asymptomatic patients, requiring further investigation. In addition, as malignant adnexal masses are usually mistaken for this condition in gynecologic practice, they should be considered in the differential diagnosis in TB-endemic regions. Definitive diagnosis is made only by histopathological and bacterial examination of biopsy tissue samples [3,4]. Identification of caseating granulomas through histopathological examination of the tissue samples is typical for diagnosis. Treatment is medical.

In this study, we aimed to investigate the incidence of pelvic TB mimicking ovarian cancer.

\section{Material and methods}

Between January 1, 2004 and January 1, 2016, data of a total of 13 patients who presented to the gynecologic oncology unit of the Kanuni Sultan Suleyman Training and Research Hospital and were diagnosed with peritoneal/genital TB were retrospectively analyzed. The medical and family history of the patients, demographic and clinical characteristics, laboratory and imaging study findings, and histopathological findings were recorded using the hospital database. In particular, demographic data including age, parity, body

Table I. Demographic, laboratory and clinical findings of women with pelvic tuberculosis

\begin{tabular}{|lcc|}
\hline Characteristics & Number $(n)$ & Rate (\%) \\
\hline Age [years] & $22-67$ & $39.4 \pm 14.1$ \\
\hline Parity & $0-5$ & $1.69 \pm 1.1$ \\
\hline Body mass index $\left[\mathrm{kg} / \mathrm{m}^{2}\right]$ & $18-43$ & $23.3 \pm 4.1$ \\
\hline CA-125 level $[\mathrm{U} / \mathrm{ml}]$ & $6-9$ & $473 \pm 394$ \\
\hline Symptoms & Number $(n)$ & Rate $(\%)$ \\
\hline Bloating & 9 & 69.23 \\
\hline Abdominal pain & 3 & 23.07 \\
\hline No symptom & 1 & 7.69 \\
\hline
\end{tabular}

mass index (BMI), history of menstrual cycle, complaints on admission, and physical examination findings were noted. Laboratory test results included CA125, complete blood count, and routine biochemistry tests. The peritoneal fluid of patients who underwent paracentesis was tested for adenosine deaminase (ADA) level, subjected to acid-fast bacilli (AFB) staining, analyzed by polymerase chain reaction (PCR) and culture for the detection of Mycobacterium tuberculosis, and cytology for the detection of atypical cells. Furthermore, radiological examination results of lung X-rays, ultrasonography (US), magnetic resonance imaging (MRI), and/or computed tomography $(\mathrm{CT})$ were recorded. The patients underwent laparotomy by umbilical medial incision or tissue sampling through laparoscopic surgery. The diagnosis was confirmed by histopathological examination. All patients received postoperative anti-TB treatment.

Written informed consent was obtained from each patient. The study protocol was approved by the local ethics committee. The study was conducted in accordance with the principles of the Declaration of Helsinki.

\section{Results}

The mean age of the patients was $39.4 \pm 14.1$ years. The most common complaints were bloating and abdominal pain (Table I). One patient had no symptoms but was referred to the gynecologic oncology unit with an adnexal mass and elevated CA125 values. There was no known medical history of TB in any of the patients; however, 4 patients were reported to have a family history of TB. Preoperative chest X-ray showed no findings of primary TB.

Table II. Pre-operative imaging findings and laboratory findings

\begin{tabular}{|c|c|c|}
\hline Characteristics & Number (n) & Rate (\%) \\
\hline \multicolumn{3}{|l|}{ USG, CT/MRI findings: } \\
\hline Ascites & 11 & 84.6 \\
\hline Pelvic mass & 10 & 76.9 \\
\hline $\begin{array}{l}\text { Omental cake, peritoneal } \\
\text { thickening }\end{array}$ & 11 & 84.6 \\
\hline X-ray & 0 & 0 \\
\hline \multicolumn{3}{|l|}{ Laboratory findings: } \\
\hline $\begin{array}{l}\text { Adenosine deaminase } \\
\text { (ADA) }\end{array}$ & $0 / 1$ & 0 \\
\hline Culture & $0 / 2$ & 0 \\
\hline ARB & $0 / 4$ & 0 \\
\hline PCR & $1 / 4$ & $25 \%$ \\
\hline
\end{tabular}


The most common US findings were diffuse intra-abdominal ascites and adnexal mass. US examination also revealed small smooth-bordered simple cystic formations of less than $4 \mathrm{~cm}$ and irregular-bordered heterogeneous complex cystic formations. Magnetic resonance imaging showed peritoneal thickening and omental cake formation (Table II).

The mean CA125 value was $473 \pm 394 \mathrm{U} / \mathrm{ml}$. In patients with no detectable adnexal mass despite findings of diffuse abdominal ascites and peritoneal thickening on US and MRI/CT, paracentesis and endoscopy of the upper and lower gastrointestinal tract were performed in 6 and 5 patients respectively to investigate the underlying etiology and confirm the diagnosis. The PCR, ADA measurement, culture, and/or cytologic examinations were performed on ascitic fluid obtained during paracentesis. Cytologic evaluation in 6 patients showed no atypical cells, and ADA assessment in 1 patient was negative. In addition, no positive results were reported from culture in 2 patients or from acid-fast bacilli (AFB) staining in 4 patients. The PCR analysis of the ascitic fluid of 5 patients yielded only one positive result. Furthermore, no findings consistent with malignancy were reported in 5 patients who underwent gastrointestinal tract endoscopy (Table II).

Laparoscopic surgery was performed in $6 \mathrm{pa}-$ tients, whereas 7 patients underwent laparotomy by umbilical medial incision (Table III). Examination of the abdomen showed diffuse pelvic and abdominal adhesions in all patients and diffuse nodular formations in pelvic and intra-abdominal organs in 11 patients. Eight patients had hydropic tubes, whereas there was no tubal pathology in the other 5 patients (Table IV). In patients with intra-abdominal ascites and diffuse nodular formations, peritoneal and omental tissue samples were obtained for diagnostic purposes and immediately sent for frozen section. Pathological examination results were benign in all patients except one. Surgical operation was terminated in patients with benign frozen sections. Subsequently, debulking surgery was performed in 1 patient diagnosed with atyp-

Table III. Surgical procedures in women with pelvic tuberculosis

\begin{tabular}{|lcc|}
\hline Surgical procedures & Number $(n)$ & Rate (\%) \\
\hline Laparoscopy & 6 & 46.15 \\
\hline Laparotomy & 7 & 53.85 \\
\hline Peroneal multiple biopsy & 9 & 69.2 \\
\hline Salpingectomy & 1 & 7.69 \\
\hline Salpingoophorectomy & 2 & 15.38 \\
\hline Debulking surgery & 1 & 7.69 \\
\hline
\end{tabular}

ical cells at frozen assessment, including total abdominal hysterectomy, bilateral salpingo-oophorectomy, omentectomy, and bilateral pelvic and para-aortic lymphadenectomy. Histopathological diagnosis was made in all patients through the identification of caseating granulomas. No complications were reported during the perioperative and postoperative periods. Postoperative anti-TB treatment was initiated in all patients.

\section{Discussion}

Tuberculosis is a bacterial infection caused by Mycobacterium tuberculosis which primarily affects the lungs, but also shows extrapulmonary involvement through hematogenous transmission [1-3]. Patients diagnosed with urogenital TB often present with complaints of irregular menses, chronic pelvic pain, or nonspecific symptoms such as bloating and agitation. Some patients are diagnosed with infertility of unknown etiology [5-7]. These cases are mostly asymptomatic [8], and are often diagnosed with pelvic TB through further testing. Another group of patients present with a pelvic mass and concomitant ascites mimicking malignant ovarian pathologies. These patients are usually referred to the gynecologic oncology department [9-11].

Radiological evaluation has demonstrated that the most common US findings are ascites and pelvic masses with omental thickening and omental cake formation on MRI and CT $[3,4]$. In a retrospective study, Koc et al. [4] reported diffuse intra-abdominal ascites in $22(100 \%)$ patients and pelvic mass in 17 (72.43\%) patients. In a similar study, Liu et al. [3] observed intra-abdominal ascites in $22(71.43 \%)$ patients, pelvic mass in 21 (75\%) patients, and omental involvement in

Table IV. Intra-operative findings of women with pelvic tuberculosis

\begin{tabular}{|lcc|}
\hline Characteristics & Number $(n)$ & Rate (\%) \\
\hline Ascites & 11 & 84.6 \\
\hline Peritoneal adhesions & 13 & 100 \\
\hline Peritoneal miliary nodules & 11 & 84.6 \\
\hline \begin{tabular}{l} 
Adnexal mass: \\
\hline \begin{tabular}{l} 
Cyst (simple) \\
\hline Serosal
\end{tabular}
\end{tabular} & 4 & 30.7 \\
\hline \begin{tabular}{l} 
None \\
\hline Serosal + cyst
\end{tabular} & 5 & 23.07 \\
\hline Tubal involvement: & 1 & 38.4 \\
\hline $\begin{array}{l}\text { None } \\
\text { Hydrosalpinx }\end{array}$ & 5 & 38.4 \\
\hline
\end{tabular}


$3(10.71 \%)$ patients. In a study conducted by Malik and Saxena [10], diffuse intra-abdominal ascites was reported in all 66 patients diagnosed with abdominal TB. Consistent with previous findings, we observed diffuse ascites in 11 (84.6\%) patients and pelvic mass in 10 (76.9\%) patients; evaluation with MRI showed omental thickening or omental cake formation in $11(84.6 \%)$ patients.

Numerous laboratory tests are used to assist diagnosis, including CA125, tuberculin skin test (PPD), M. tuberculosis culture of ascitic fluid, PCR amplification of the ascitic fluid, and AFB and ADA tests. Several studies have demonstrated a significant increase in CA125 levels in ovarian malignancies, as well as in cases with peritoneal TB [2-4]. Similarly, the mean CA125 serum level was found to be $473 \pm 394 \mathrm{U} / \mathrm{ml}$ in our study.

In TB-endemic regions, differential diagnosis to exclude TB can be made through the evaluation of culture samples, PCR amplification test, ARB or ADA, using the paracentesis and ascitic fluid. The sensitivity of these procedures ranges from $80 \%$ to $93 \%$ [11]. Mycobacterium tuberculosis cultures are variably positive (20\% to $83 \%)$ $[9,10]$. In 2 patients diagnosed with active pulmonary TB by Koc et al. [4], paracentesis was performed to rule out malignancy and diagnose peritoneal TB; however, a diagnosis of peritoneal TB could not be established using this approach [4]. Liu et al. [3] reported that AFB was negative in 20 patients tested, TB culture and PCR tests were negative in 2 patients tested, while the ADA was positive in all 3 patients tested. In the aforementioned study, cytologic examination of ascitic fluid revealed no malignancies. In our study, we obtained negative results for ADA in 1 patient assessed, culture in 2 patients, and ARB test in 4 patients. Of the 4 patients we evaluated with $P C R$, only 1 was positive.

While imaging modalities such as chest $x$-ray and $\mathrm{CT}$ facilitate the diagnosis of primary pulmonary TB, abdominopelvic US, CT, positron emission CT (PET-CT), and MRI are used for the diagnosis of peritoneal or urogenital TB [10, 11, 14-16]. In addition, the presence of pleural effusion, nodularity, pleural thickening, pathological mediastinal lymph nodes, or thoracic vertebral involvement is also useful in the evaluation of TB. Abdominopelvic US, CT, or MRI may also play an important role in the differential diagnosis with findings such as adnexal masses and concomitant ascites or omental thickening, and the presence of positive lymph nodes [3, 4, 7, 14].

Although the aforementioned tests are helpful for the differential diagnosis, a definitive diagnosis of TB is only made by histopathological examination of tissue samples [3, 4, 12, 15]. Lap- aroscopy is a convenient and safe procedure for tissue sampling in patients with suspected peritoneal or genital TB. In our study, histopathological examination showed caseating granulomas in all patients. Postoperative anti-TB therapy was administered for a period of 6 months.

Urogenital/peritoneal TB is usually confused with malignant adnexal masses due to the similar nature of the symptoms. Peritoneal or urogenital TB should definitely be considered in the differential diagnosis of malignant adnexal masses, particularly in TB-endemic regions. Preoperative laboratory tests are also helpful in the diagnosis; however, histopathological and bacterial examination of tissue samples is essential for definitive diagnosis.

\section{Conflict of interest}

The authors declare no conflict of interest.

\section{References}

1. Chiang CY, Centis R, Migliori GB. Drug-resistant tuberculosis: past, present, future. Respirology 2010; 15: 413-32.

2. Masiello A, Pacifico P, Giglio S, et al. [Abdominal tuberculosis in a young immigrant patient: a clinical case]. Infez Med 2012; 20: 120-4.

3. Liu Q, Zhang Q, Guan Q, Xu JF, Shi QL. Abdominopelvic tuberculosis mimicking advanced ovarian cancer and pelvic inflammatory disease: a series of 28 female cases. Arch Gynecol Obstet 2014; 289: 623-9.

4. Koc S, Beydilli G, Tulunay G, et al. Peritoneal tuberculosis mimicking advanced ovarian cancer: a retrospective review of 22 cases. Gynecol Oncol 2006; 103: 565-9.

5. Devi L, Tandon R, Goel P, et al. Pelvic tuberculosis mimicking advanced ovarian malignancy. Trop Doct 2012; 42: 144-6.

6. Koc S, Beydilli G, Tulunay G, et al. Peritoneal tuberculosis mimicking advanced ovarian cancer: a retrospective review of 22 cases. Gynecol Oncol 2006; 103: 565-9.

7. Wu CH, Changchien CC, Tseng CW, et al. Disseminated peritoneal tuberculosis simulating advanced ovarian cancer: a retrospective study of 17 cases. Taiwan J Obstet Gynecol 2011; 50: 292-6.

8. Crochet JR, Hawkins KC, Holland DP, Copland SD. Diagnosis of pelvic tuberculosis in a patient with tubal infertility. Fertil Steril 2011; 95: 289 e17-20.

9. Sharma JB, Sneha J, Singh UB, et al. Comparative study of laparoscopic abdominopelvic and fallopian tube findings before and after antitubercular therapy in female genital tuberculosis with infertility. J Minim Invasive Gynecol 2016; 23: 215-22.

10. Malik A, Saxena NC. Ultrasound in abdominal tuberculosis. Abdom Imaging 2003; 28: 574-9.

11. Vardareli E, Kebapci M, Saricam T, Pasaoglu O, Acikalin M. Tuberculous peritonitis of the wet ascitic type: clinical features and diagnostic value of image-guided peritoneal biopsy. Dig Liver Dis 2004; 36: 199-204.

12. Piura B, Rabinovich A, Leron E, Yanai-Inbar I, Mazor M. Peritoneal tuberculosis mimicking ovarian carcinoma with ascites and elevated serum CA-125: case report and review of literature. Eur I Gynaecol Oncol 2002; 23: 120-2. 
13. Straughn JM, Robertson MW, Partridge EE. A patient presenting with a pelvic mass, elevated CA-125, and fever. Gynecol Oncol 2000; 77: 471-2.

14. Jeffry L, Kerrou K, Camatte $S$, et al. Peritoneal tuberculosis revealed by carcinomatosis on CT scan and uptake at FDG-PET. BJOG 2003; 110: 1129-31.

15. Ertas IE, Gungorduk K, Ozdemir A, et al. Pelvic tuberculosis, echinococcosis, and actinomycosis: great imitators of ovarian cancer. Aust N Z J Obstet Gynaecol 2014; 54 : 166-71.

16. Kim SH, Yang DM, Kim KA. Unusual causes of tubo-ovarian abscess: $C T$ and MR imaging findings. Radiographics 2004; 24: 1575-89. 